\title{
Autonomy in Physical Human-Robot Interaction: a Brief Survey
}

\author{
Mario Selvaggio $^{1}$, Marco Cognetti ${ }^{2}$, Stefanos Nikolaidis ${ }^{3}$, Serena Ivaldi ${ }^{4}$, and Bruno Siciliano ${ }^{1}$
}

\begin{abstract}
Sharing the control of a robotic system with an autonomous controller allows a human to reduce his/her cognitive and physical workload during the execution of a task. In recent years, the development of inference and learning techniques has widened the spectrum of applications of shared control (SC) approaches, leading to robotic systems that are capable of seamless adaptation of their autonomy level. In this perspective, shared autonomy (SA) can be defined as the design paradigm that enables this adapting behavior of the robotic system.

This letter collects the latest results achieved by the research community in the field of SC and SA with special emphasis on physical human-robot interaction (pHRI). Architectures and methods developed for SC and SA are discussed throughout the paper, highlighting the key aspects of each methodology. A discussion about open issues concludes this letter.
\end{abstract}

Index Terms-Physical Human-Robot Interaction; HumanCentered Robotics; Human-Robot Collaboration.

\section{INTRODUCTION}

A FULLY autonomous robot is a machine that is able to carry out a task by sensing, planning, and acting into an environment without any human intervention. However, despite the great progress achieved by automation in the recent years, we are still far from providing robots with full autonomy, that would allow them to successfully deal with unpredictable events or unforeseen situations. Nowadays, in most robotic applications, it is customary to have robots that are operated or supervised by a human operator, who can provide superior situation awareness, logic, and problem-solving capability. Sometimes, this is enforced by safety regulations and/or ethical concerns (e.g., a robot is still fully operated by a doctor during surgical procedures). In other words, the interaction between humans and even the most autonomous robot, albeit minimal, is needed and often desirable.

In Human-Robot Interaction (HRI) applications, autonomy constitutes a means rather than the goal and its level varies

Manuscript received: February 24, 2021; Revised: June 1, 2021; Accepted: July 19, 2021.

This paper was recommended for publication by Editor Jee-Hwan Ryu upon evaluation of the Associate Editor and Reviewers' comments. This work was supported by the European Union's Horizon 2020 research and innovation programme under grant agreement No 101017008 .

${ }^{1}$ M. Selvaggio and B. Siciliano are with the Department of Electrical Engineering and Information Technology, University of Naples Federico II, Naples, Italy. Corresponding author e-mail: \{mario.selvaggio\}@unina.it

${ }^{2} \mathrm{M}$. Cognetti is with the Department of Electronic Engineering, National University of Ireland Maynooth, Maynooth, Ireland.

${ }^{3} \mathrm{~S}$. Nikolaidis is with the Department of Computer Science, University of Southern California, Los Angeles, USA.

${ }^{4} \mathrm{~S}$. Ivaldi is with the Inria, Loria, Université de Lorraine, CNRS, Nancy, France.

Digital Object Identifier (DOI): see top of this page. widely from one application to another. Autonomy allows reducing the human operator's workload when performing a task that can be repetitive and/or requires skills, effort or precision levels that exceed those of a human.

In the past, several robot control architectures were designed for humans to interact with a (partially) autonomous robot. This design methodology was typically referred to as Shared Control (SC). In the last decade, the advancements of sensing, inference, modeling, and learning methods have extended SC capabilities and have widened its spectrum of applications. This gave rise to Shared Autonomy (SA) approaches where the robot is capable of seamless adaptation of its autonomy level based on its own understanding of the human actions/intentions and of the surrounding environment. Adaptation is the most desirable feature when the robot operates in dynamically changing environments, needs to accomplish diverse tasks and/or when the human behavior evolves over time. The autonomy level is typically adapted by opportunely arbitrating the user and the autonomous controller inputs. Until now, SC and SA expressions have been used interchangeably. In this survey, we make this distinction clearer: in SA the robotic system automatically adjusts its level of autonomy based on internal/external information, while in SC the human manually tunes it. The application determines the paradigm that needs to be adopted.

In both approaches, autonomy constitutes the key aspect to be properly designed. In the past, other papers tried to propose continuous or discrete classifications of autonomy levels in SC applications. However, these classifications are drawn for specific domains such as telerobotics [1], [2], autonomous vehicles [3], [4], and surgical robotics [5], and are hard to be generalized. In general, it would be more appropriate to define a spectrum in place of discrete levels of autonomy for SA approaches, since the robot may continuously vary its autonomy level in function of external signals coming from the human, the task, and/or the environment.

In this survey, we review the use of autonomy in physical Human-Robot Interaction (pHRI). For us, the word physical comprises both proximal and remote (bilateral teleoperation) HRI scenarios. In the former, the human and the robot are in direct contact, which may be mediated by a third object (e.g., in cooperative manipulation scenarios). In the latter, the connection between the human, at the local site, and the robot, operating in a remote environment, is obtained by means of an appropriate force feedback action that is provided to the user. This survey provides the following contributions: (i) we review the role of autonomy in pHRI, highlighting the difference between SC and SA as design paradigms; 


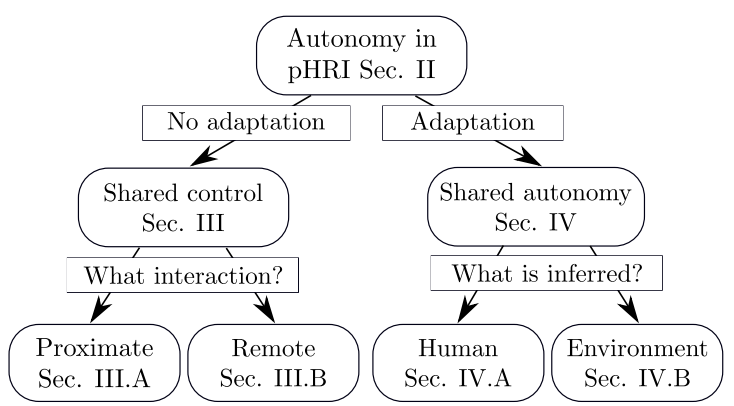

Fig. 1. Categorization of approaches considered in this brief survey.

(ii) we classify the most recent papers proposing SC and SA approaches; (iii) we collect and discuss open challenges and future research directions.

This survey is not meant to be a comprehensive literature review, rather, we want to provide the reader with an overview of the different approaches. A recent literature review on intention detection and arbitration for pHRI is given in [6]. To the best of our knowledge, the considered work is the closest to this survey. However, it mostly focused on medical and rehabilitation fields while our focus comprises a broader range of systems and applications.

The rest of the paper is organized as shown in Fig. 1: Section II overviews the general concepts behind the use of autonomy in pHRI systems; Section III reviews the state-ofthe-art SC methods while Section IV overviews the latest SA methods. In addition, Section V discusses haptic-based communication methods in SC/SA while Section VI concludes the paper by discussing open challenges.

\section{AUTONOMY IN PHYSICAL HUMAN-ROBOT INTERACTION}

In a very broad sense, autonomy can be integrated in a pHRI scenario as shown in Fig. 2. The evolution of the robotic system can be described by the following dynamics:

$$
\begin{aligned}
& \dot{x}(t)=f(x(t), u(t)) \\
& u(t)=h_{\theta}\left(u_{h}(t), u_{a}(t) ; \theta(t)\right),
\end{aligned}
$$

where $x$ is the robot/environment state, $u$ is the control input. A (possibly non-linear) arbitration function $h_{\theta}$ combines/modulates $u_{a}$ and $u_{h}$, which are the autonomous control and the human inputs, respectively. Modulating the two inputs $u_{h}$ and $u_{a}, h_{\theta}$ determines the autonomy level of the robotic system. Here, $\theta$ models the robot understandings of the human and/or of the environment used by the arbitration function to modulate the two inputs. For example, $\theta$ may contain information about the human action/intention or the task completion status. In general, $u_{h}$ is a signal belonging to the following robot channels: configuration, inputs and/or task.

A SA system modifies the weight of the human and the autonomous control inputs based on $\theta$. Conversely, in SC the arbitration function $h_{\theta}$ reduces to $h\left(u_{h}, u_{a}\right)$, i.e., it does not depend on external variables other than the human/autonomous control inputs. This function is totally designed by the human - usually to be fixed during a task - resulting in a user-defined role/autonomy division between the user and the autonomous

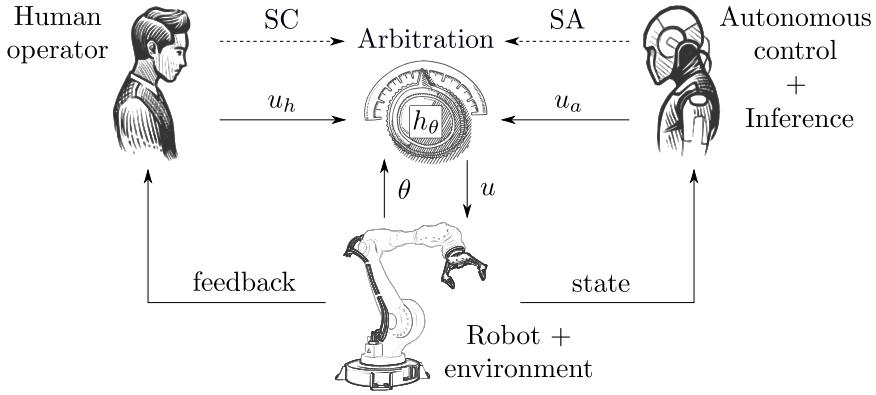

Fig. 2. A general architecture integrating autonomy in a pHRI scenario. Symbols are explained in Sec. II. Arbitration $\left(h_{\theta}\right)$ of the control signals $\left(u_{h}, u_{a}\right)$ is either tuned by the human operator - shared control (SC) - or by the autonomous control through inference - shared autonomy (SA).

controller. As matter of fact, no system intelligence is involved, and the robot does not perform any adaptation when the human/environment evolve their behavior/status over time. To give an example, most of the times SC approaches are designed to implement a linear combination of the human and the autonomous controller signals, i.e., (in the scalar case)

$$
h\left(u_{h}, u_{a}\right)=\alpha u_{h}+(1-\alpha) u_{a}
$$

where $\alpha \in[0,1]$ is a weight allocating the control authority between the human and the autonomous controller. In a SC approach, the weight $\alpha$, or more in general the arbitration function $h$, is designed by the human, which may also manually modify/tune it over time. In case $\alpha$ is automatically tuned, e.g., through inference of the human actions [7], the same arbitration function can be used to realize a SA system.

\section{SHARED CONTROL}

In this section, we review SC approaches and classify them based on the type of interaction: proximate (Sec. III-A) and remote (Sec. III-B).

SC was primarily introduced as a control architecture for remotely operated robots where embedding some autonomy in the robot was essential to overcome large communication delays between the local and remote sites [8]. Classically, the architectures corresponding to different human interaction modalities have been grouped into three classes: 1) direct control, which implies no intelligence or autonomy in the system, all the degrees-of-freedoms (DoFs) of the robot are directly controlled by the user via local interfaces; 2) supervisory control, where the user commands and feedback occur at a higher level, the connection is looser and the robot has to rely on a stronger local autonomy to refine and execute tasks [9], [10]; 3) shared control, comprehensive of all the intermediate levels in which the robot is controlled by a combination of direct user commands and autonomy [11]. In this context, the most preeminent classification of autonomy levels was proposed in [12].

Generally, a SC architecture is conceived to provide motion commands correction/overlay or assume control of subtasks. For instance, motion overlay compensating beating hearth movements during robotic surgery gives the user the possibility of operating on a virtually stabilized patient [13]. Subtask control is used to maintain a stable grasp over long time periods 
relieving the user from constantly imposing the corresponding commands [14]. In general, SC paradigms are designed to combine the cognitive skills of the human and the robustness and precision abilities of robots.

This design paradigm evolved in the context of telerobotics and found numerous applications in diverse contexts, such as space/undersea exploration [15], [16], wheelchairs control [17], autonomous driving [18], humanoid teleoperation [19], nuclear sites decommissioning [20], aerial robotics [21], assistive robotics [22], and surgical robotics [23].

In most of the above-mentioned contexts, the human usually interacts with a complex robotic system (e.g., having many DoFs) to accomplish one or more tasks simultaneously. SC is, thus, required to reduce the amount of user's workload that would be necessary to direct control the system. For example, controlling the rotational end-effector DoFs of a robotic arm contributes significantly more to decreased performance and increased difficulty than translational ones [24]. SC may be designed to reduce the effect of these difficult-to-control features on task performance [25].

\section{A. Proximate interaction}

In the context of proximate pHRI, SC principles are leveraged to design collaborative [26] and cooperative robotic systems [27] integrating autonomy. For instance, SC methods are used to enable cooperative manipulation of long objects (grasped at the two ends by the human and the robot) constraining their motion as they were transported on a wheel [28] or varying the task effort assignment via dynamic role exchange/sharing mechanisms [29]. In this case, SC can be implemented through classical or variable impedance/admittance control techniques [30], virtual constraints [31], or hybrid force/velocity control [32]. An admittance-based SC architecture for collaborative object transportation with dual-arm robot is developed in [33] to increase the productivity in manufacturing: estimating and compensating the object gravity is performed autonomously, while human applied force in the cooperative task space is used to move the object in the desired direction. Similarly, a group of mobile robots can transport an object in cooperation with a human by compensating its dynamics and orienting towards the user-preferred direction of motion acting as Caster-like wheels [34]. Outside industrial environments, cooperative object manipulation requires constraint avoidance algorithms and effort sharing policies [35]. Recent work is focusing on designing distributed multi-robot SC frameworks where a human operator physically interacts with an object manipulated by a multi-manipulator robotic system [36].

Semi-autonomous driving, in which a user and an autonomous controller simultaneously control the vehicle, is another field in which proximate interaction SC is highly employed. Shared control of intelligent vehicles between a human driver and a lane-keeping and obstacle avoidance assisting system is addressed in [37]: the steering assistance actions are computed according to the driver's real-time driving activity. Finally, SC is leveraged for wheelchair control in tight environments in [38]: the driver commands a preferred velocity which is transformed into a collision-free smooth local motion that respects the actuator constraints. Although SC allows the human to always takeover in case of disagreement with autonomy, it lacks the additional intelligence that can be provided by estimation of human intentions, desires, and beliefs as in SA.

\section{B. Remote interaction}

In remote pHRI scenarios, the human and the robot are spatially separated, and the interaction is established through appropriately designed communication channels. SC methods are used to implement a task- or a trajectory-level interaction.

The former approach is used when accomplishing a task requires fulfilling several sub-tasks simultaneously. Thus, the accomplishment of some sub-tasks is carried out by the autonomous controller to facilitate the human operator. For instance, an autonomous control action makes the robot gripper always pointing in the direction of an object to facilitate its approaching and grasping during sort and segregation of nuclear waste activities [25]. Task prioritization and wholebody control architectures allows implementing a hierarchical division of roles between the human and the autonomy [39]: this allows a humanoid robot to coordinately accomplish manipulation, locomotion and constraint avoiding tasks while accepting position goals issued by a human [16], [40].

The latter approach, allows the user to interact in real-time with a remote inspection robot at the trajectory level: the user can steer the reference path of an autonomous mobile robot (e.g., a UAV) by acting on path parameters that are simultaneously affected by an autonomous algorithm to ensure collision avoidance, path regularity, proximity to points of interest [41] or maximize the collected environmental information [42].

Besides, SC can be designed to improve the user's ability to remotely operate complex machines while simultaneously avoiding unsafe regions [43]: to this end, obstacles avoidance can be performed by autonomously overriding the user's commands leveraging reactive techniques such as artificial potentials fields [16] or model predictive control [44].

Dual-arm robotic systems are typically employed to grasp and manipulate large objects: SC allows a human operator to specify the object motion while autonomy performs coordinated control of the arms to realize grasp stability in the remote space [45], [11]. A framework to accomplish both coordinated bimanual grasping and asymmetric tasks is proposed in [46], while a SC architecture with a dynamic selection of the most suitable robot to be commanded by the user is presented in [47]. Frequently, SC for dual-arm systems is designed with one arm remotely operated and the other completely autonomous and equipped with an eye-in-hand camera to provide visual feedback. In this case, a vision-based autonomous controller allows the execution of occlusion-free tasks [48], while avoiding self-collisions, joint limits, and singularities constraints [49].

The benefits of SC on the human operator's workload are even more evident when a group of mobile robots (e.g., UAVs) need to be controlled by a user as in real-world search and rescue missions. SC architectures providing connectivity 
maintenance, formation control, and obstacle avoidance functionalities allow the user to control a semi-autonomous group of UAVs while redeeming from directly commanding single agents [50], [51], [21]. More recently, SC was used to perform cooperative grasping and transportation of an object with a group of aerial manipulations [52], [53] or with heterogeneous robot teams [54] following the operator's commands. In a recent and comprehensive review on SC methodologies for human-robot team interaction, the autonomy level adaptation, e.g., based on the human confidence in performing certain tasks, is identified as a key step to be taken in the future [55].

\section{Shared AUTONOMY}

As discussed above, in SC approaches the amount of autonomy, shared between the human and the robot, is either static or manually tuned by the human. However, as the physical interaction between the human and the robot has an evolving nature, autonomy adaptation approaches were started to be devised paving the way towards SA architectures. This enabled a human-robot interaction much closer to a human-human one where (mutual) adaptation is a predominant aspect.

The transition from the SC to the SA paradigm was smooth. Some works preliminary introduced human-robot cooperative control with different autonomy levels leveraging features such as active constraints, machine learning, and automated movements in the context of robotic surgery [56]. On this line, platforms able to deal with different autonomy levels for human-robot coexistence and collaboration in manufacturing applications were developed [57]. Besides surgical and industrial scenarios, several other fields envisioned the use of multilevel autonomy such as autonomous driving [18], space [58], and assistive robotics [22].

As stated above, in the SA paradigm the autonomy level is dynamic and its adaptation is seamlessly performed by the robot during the task execution. This adaptation is usually performed leveraging information extracted from the human and/or from the environment (including the task). According to the source of information that triggers the autonomy adaptation, we list approaches that extract information from the human in Sec. IV-A and approaches that use environmental information in Sec. IV-B.

\section{A. Human}

In this section, we survey approaches that extract and use information from the human operator to adapt the autonomy. In the following, we consider human intentions (Sec. IV-A1), muscle activity (Sec. IV-A2), and skills (Sec. IV-A3).

1) Human intentions: A SA system may infer which is, in a probabilistic sense, the action that the human is performing. Based on this information, it can compute what action the robot must undertake, regulating its autonomy and/or providing assistance. Leveraging this concept, a human-robot mutual adaptation framework for collaborative object transportation tasks is presented in [59], [60]: a bounded memory adaptation model assumes that the human stochastically switches between a finite set of modal policies and a mixed-observability Markov decision process chooses the robot action, accordingly. A SA approach for assistive teleoperated robots that infers and predicts human intentions is proposed in [7]: a recursive Bayesian filter fuses multiple observations to infer the human goal and alter the system autonomy level in function of its estimated uncertainty. On the same line, a framework based on policy blending is presented in [61]: the robot assistance, during a teleoperated reach-to-grasp task execution, is provided as arbitration of two policies, namely, user's input and the robot's prediction of the user's intent.

Approaches based on SA can be used to assist humans with disabilities in object reaching and manipulation (e.g., slicing and scooping) tasks: a linear blending function with user-tunable parameters and a confidence metric estimating the human goal are used in [62] to determine the arbitration between the autonomous controller and the human. Similarly, the human task is modelled as a Markov decision process in [63], which provides the autonomous controller with the human goal estimates using dimensionality reduction techniques. A SA teleoperation framework, which mimics the arm movements of an operator through a bimanual robotic system, is presented in [64]: the human is assisted on-the-fly in completing different bimanual tasks (e.g., object handover, container opening) by means of a recurrent neural network using the user motion information detected through a motion capture system. Gametheoretic approaches also can be used to build SA frameworks: the system assumes that the human is optimizing its objective function, while its latent intention is inferred from feedback errors to determine the parameters of a variable impedance controller during human-robot co-assembly tasks [65], [66].

2) Human muscle activity: Instead of inferring the human intentions, some papers focus on estimating a metric related to the human fatigue and take actions for minimizing it. A framework for proximal human-robot collaboration is presented in [67]: the robot implements a hybrid force/impedance controller and estimates the human muscle activity while performing the task thanks to wearable EMG electrodes. When the human muscle activity exceeds a threshold, the robot uses the learned skills (encoded as periodic dynamic motion primitives) for helping the human in reducing his/her fatigue. A similar idea is proposed in [68], where the 'rapid upper limb assessment' is used to estimate the human muscle activity during remote teleoperation tasks: based on this information, the robot adapts its trajectory to minimize it. An interesting emerging direction consists in estimating the human fatigue thanks to a continuous monitoring of the human activities [69].

3) Human skills: Attributing skills to humans allows defining distance metrics between the performances of expert and naive operators. The autonomy level can then be autonomously regulated according to these metrics. In surgical robotics, a SA teleoperation framework that adapts its cooperative properties to the estimated skill level of the operator is proposed in [70]: skill profiles, captured as task performance measures, are exploited to modify the behavior of the assistive robotic system and enhance the user experience by preventing unnecessary restrictions for skilled users. On the same line, the assistanceas-needed paradigm provides the user with variable assistance during surgical training according to its current and past performances [71]. A partially observable Markov decision 
process is used to represent the expertise level of an operator during teleoperated navigation of a mobile robot [72]: the SA framework uses the inferred user's expertise level and environmental observation to fuse the user's input with the appropriate autonomous controller.

In general, the correct interpretation of data and attribution of human mental states are crucial aspects in SA systems. Perspective-taking (i.e., the ability to take one another's perspective and reason from this alternative point of view) on robots would be a valuable asset for people working with them [73]: interpreting data from a human perspective allows overcoming ambiguity and incompleteness that can often be present in human demonstrations [74] and communicate and interact naturally with humans [75]. Theory of mind computational models allow attributing beliefs, goals, and desires to the human operator [76], [77]. In this perspective, neural networks have shown to be capable of building models for agents' behavior predicting the main characteristics and mental states [78]. However, the development of efficient computational models that predict the human behavior in realtime is still an open challenge as will be discussed in Sec. VI.

\section{B. Environment}

In this section, approaches that use information about the environment (rather than the human operator) to adapt the autonomy level are discussed. The most common method to retrieve environmental information is to focus on what the robot has to fulfill, i.e., the task, and extract information directly from it. In this perspective, Learning-from-Demonstration (LfD) has been proven to be an effective approach: the idea is to extract expert users' behavior while executing some tasks, and use this information to help non-expert users in accomplishing similar tasks. This paradigm is adopted in the context of a pick-and-place remote manipulation task in [79], where trajectories from expert users are captured through a haptic device, encoded into some distributions, and then used by an adaptive controller to determine the system autonomy. This way, naive operators are assisted during the task execution through haptic cues that point in the direction of the trajectories performed by the expert users.

Similarly, a task-parametrized Gaussian Mixture Model (GMM) is used to build a representation of a remotely executed scanning task in [80]: datapoints from expert operators are collected to encode the task. This is used, together with the user input, inside a linear quadratic regulator that computes the robot stiffness and damping that determines the autonomy level of the teleoperated robot. A similar approach is presented in [81], where a Task-Parametrized Hidden Semi-Markov Model (TP-HSMM) is used together with an incremental online learning algorithm to encode models of a hot-stabbing task. A model predictive control, that uses the TP-HSMM predicted state evolution, allows anticipating and adapting the robot to future events. The approach is extended in [82], where the system is also able to automatically disambiguate between local and remote task parametrizations. LfD is used in [83] for encoding remotely performed peg-in-hole tasks: GMMs are used to extract information from demonstrations of expert operators, which is then used to generate forcebased haptic guidance trajectories that help non-expert users in fulfilling the insertion task. Similarly, LfD is used to create surgical task models in [56]: the proposed framework collects demonstrations and segments the task based on tool-tissue or tool-tool interaction. The task is encoded as a continuous hidden Markov model which is used to regress a path among those captured during the demonstration, helping naive users through visual and haptic cues.

Besides the task, environmental constraints can be used to trigger the system adaptation. An adaptive authority framework handling target occlusions is presented in [84]: the control allocation between the human and the autonomous controller is adapted based on target measurements uncertainty of an adaptive Bayesian filter. This estimates the pose of the target based on visual measurements and its covariance matrix is used to linearly weight the contribution of the human and the autonomous controller.

It is worth to note that the use of human/environment sources of information is not mutually exclusive. A challenge in the design of effective SA frameworks is to understand how to properly combine and, possibly, prioritize information gathered from multiple sources, as will be discussed in Sec. VI.

\section{HAPTIC COMMUNICATION}

In both remote and proximate pHRI scenarios, haptic communication between the human and the robot is always inherently present. In some SC/SA approaches the autonomous controller inputs, opportunely arbitrated, are provided to the users as haptic signals [79]. Control of the robotic system is thus shared, since user's movements are influenced by haptic signals generated exploiting knowledge of the environment and the task [85]. In this way, the potentially variable autonomy is reflected to the user, the system becomes more legible/transparent, while the operator retains its full control.

The exchange of forces helps to increase the human situation awareness providing useful information about, e.g., the current system status, proximity to constraints [39], environmental obstacles [86], etc. Haptic signals integrated in SC/SA systems can be equivalently seen as virtual fixtures, that help the human to operate into restricted regions and/or to move along desired paths [87], [31]. Haptic displays realize the cobotic behaviour of the robotic device [26] making it potentially well-suited to safety-critical tasks (e.g., surgery) [88]. However, their effective integration is subject to the stability and the safety certification of the interactive robotic system. Haptic-based SC/SA frameworks have been developed to guide the user towards grasp poses that maximize manipulation capabilities [89], [90], or avoid incurring into the system constraints along post-grasping trajectories [91]. At a larger scale, whole-body haptic teleoperation interfaces are currently being developed for bipedal robots to provide the user with a sense of the robot's executed motion [92].

Contextualized haptic assistance can be provided to the operator in a structured manner exploiting models of the task learned from demonstration [82], [56]. The level of autonomy, adjusted exploiting the confidence into human and autonomous 
control, is reflected to the user through haptic guidance [93], [80]. Exploiting the online estimated probability of following a certain trajectory, mechanisms for automatic regulation of haptic assistance have been developed [94], [95]. However, the haptic feedback provided to the human operator may be in conflict with other sensory signals (e.g., audio or visual). The conflict may be caused by a low confidence estimation of human goals performed by the autonomous control. This results in low or wrong assistance causing a decrease of trust in automation by the human operator [96], [97]. Accounting for this in the design of SA frameworks can potentially lead to reduced user's workload and higher trust in automation [98] as discussed in the next section.

\section{CONCLUSIONS AND DISCUSSION}

In this letter, we briefly surveyed the use of autonomy in pHRI, discussing SC and SA as implementation paradigms. The autonomy level is manually designed and tuned by the human in SC while it is automatically adapted by the system in SA exploiting the robot understanding of the human and/or the task/environment.

In this view, SC still constitutes a powerful paradigm for combining human decision-making and robot precision capabilities. Indeed, it provides assistance through commands overlay and subtasks control while leaving the human operator the ultimate authority over the system. The common trend behind the design of SC architectures is the leverage of task models and model-based control. However, while this is advantageous for stability and safety certification, it makes SC strategies specific to the application domain and to the task to be performed. As no autonomy adaptation is envisioned in this paradigm, any change in the task requires substantial strategy modifications leading to tedious interruptions and setting up times. This constitutes the main limiting factor, as the level of the robot autonomy is desirable to change according to the human/environment evolving behavior.

Determining the level of autonomy that a human wants a robotic system to have is a very complex problem. There are cases in which users appreciate that the robotic system guides them [99]. However, there are also cases where the user wants that the autonomy is limited and the robotic system does not take invasive actions, (see, e.g., the surgical domain). Since pHRI embraces a wide range of applications, we believe there is no unique answer to this open problem but it requires the development of a case-by-case solution.

Contrarily, SA approaches provide this favorable autonomy level adaptation feature that leverages inference of the human intentions or the task progress. However, other environmental information are typically ignored. Thus, some of the open questions are: how does this information increase the versatility of the robots in performing everyday tasks? How to combine the information coming from multiple sources in a unified framework? Our intuition is that the SA framework should be able to dynamically understand the importance of each source and appropriately choose its autonomy level.

As advanced SA techniques are being developed, using various adaptation mechanisms and haptic communication means, these require novel control methodologies that assure a safe interaction between the human and the robotic system. The enhanced flexibility of SA systems, that are capable of providing contextual or personalized assistance and seamless adaption of the autonomy level, is a desirable trend that raises new challenges for safety and stability certification [100]. A possible approach is to use passivity-based control techniques as largely done for SC scenarios [101].

As discussed in Sec. IV, most of the SA approaches use user's goals inference within a predict-then-act paradigm. However, when the user's goals cannot be predicted with high confidence, SA methods may not assist the user or give little assistance [96]. Developing reliable inference methods to effectively regulate assistance is still an open research field. Moreover, SA approaches that require task/environment inference are challenged by unstructured environments with ill-defined tasks. This issue may be mitigated by developing approaches that render robots capable of online learning, updating in real-time their understandings of the user's goals and of the task/environment. To this end, reinforcement learning and deep learning techniques are capable of learning autonomy/assistive policies directly, instead of optimizing them. In particular, deep reinforcement learning allows implementing model-free SA but this typically requires lots of training data, which can be burdensome for human users operating physical robots [102].

The concept of human trust in autonomy is another essential aspect to be considered when developing effective humanrobot collaboration techniques. Trust favors the adoption of semi-autonomous systems such as robot assistants [97]. SA paradigms integrating the notion of trust require the definition of its computational model, which is a research question per $s e$. The trust towards a robotic system depends on many factors such as the context, the application, and several individual factors such as user's attitude and experience. It also evolves over time, which can be anchored in specific features of the robot, and can be influenced by the user experience and the robot behavior [103]. Developing sophisticated predictive models integrating trust into the robot decision-making in a principled way, while maintaining computational tractability, is an exciting area for future work [60]. This opens interesting questions: how much autonomy should the system have? How does this affect users' trust and their willingness to use the system? Transparency and explainability of the system behavior, intended as the human understanding of what the system is doing, why, and what it will do next is another important aspect to consider [104]. In this context, haptics may be used to increase system legibility and the situational awareness of the human, which may increase trust towards the system.

Ultimately, the evaluation of SA systems is highly subjective, users generally may prefer more assistance when performing difficult tasks, as this might allow a more efficient task completion. In our opinion, SA approaches require the development of contextualized benchmarking and validation methods for their objective evaluation. These validations in simulated and/or real-world scenarios may speed up the deployment of robots in our every-day life [105]. 
Solving the above-mentioned open issues will bring to a significant advancement in the field of pHRI. We believe that SA will be a fundamental paradigm in the next future, especially for helping non-experts and people with disabilities in performing everyday tasks, thanks to its nature to adapt to the user's requirements.

\section{REFERENCES}

[1] W. R. Ferrell et al., "Supervisory control of remote manipulation," IEEE Spectrum, vol. 4, no. 10, pp. 81-88, 1967.

[2] T. Sheridan et al., "Human and computer control of undersea teleoperators," 121978.

[3] S. Thrun, "Toward robotic cars," Communication of the ACM, vol. 53, no. 4, pp. 99-106, Apr. 2010.

[4] "Taxonomy and definitions for terms related to on-road motor vehicle automated driving systems," 2016, [Accessed 24-Feb-2021]. [Online]. Available: https://www.sae.org/standards/content/j3016_201806/

[5] G.-Z. Yang et al., "Medical robotics-regulatory, ethical, and legal considerations for increasing levels of autonomy," Sci. Robot., vol. 2 , no. 4, 2017.

[6] D. P. Losey et al., "A Review of Intent Detection, Arbitration, and Communication Aspects of Shared Control for Physical Human-Robot Interaction," Appl. Mech. Rev., vol. 70, no. 1, 022018.

[7] S. Jain et al., "Probabilistic human intent recognition for shared autonomy in assistive robotics," ACM Trans. Hum.-Robot Interact., vol. 9, no. 1, 2019.

[8] G. Niemeyer et al., Telerobotics. Cham: Springer International Publishing, 2016, pp. 1085-1108.

[9] J. H. Park et al., "Supervisory teleoperation control using computer graphics," in Proc. IEEE Int. Conf. Robot. Autom., vol. 1, 1991, pp. 493-498.

[10] T. Blackmon et al., "Model-based supervisory control in telerobotics," Presence: Teleoperators and Virtual Environments, vol. 5, pp. 205-223, 1996.

[11] R. L. Williams et al., "Shared control of multiple-manipulator, sensorbased telerobotic systems," in Proc. Int. Conf. Robot. Autom., vol. 2, 1997, pp. 962-967 vol.2.

[12] T. B. Sheridan, Humans and automation: System design and research issues. Human Factors and Ergonomics Society, 2002.

[13] T. Ortmaier et al., "Motion estimation in beating heart surgery," IEEE Trans. Biomed. Eng., vol. 52, no. 10, pp. 1729-1740, 2005.

[14] W. B. Griffin et al., "Feedback strategies for telemanipulation with shared control of object handling forces," Presence, vol. 14, no. 6, pp. 720-731, 2005.

[15] A. Douglas et al., "Real-time shared control system for space telerobotics," J. Intell. Robot. Syst., vol. 13, no. 3, pp. 247-262, 1995.

[16] G. Brantner et al., "Controlling ocean one: Human-robot collaboration for deep-sea manipulation," J. Field Robot., vol. 38, no. 1, pp. 28-51, 2021

[17] A. V. Nguyen et al., "Shared control strategies for human-machine interface in an intelligent wheelchair," in IEEE Int. Conf. Engineering in Medicine and Biology Society, 2013, pp. 3638-3641.

[18] M. Johns et al., "Exploring shared control in automated driving," in Proc. ACM/IEEE Int. Conf. on Hum.-Robot Interact., 2016, pp. 91-98.

[19] L. Penco et al., "A multimode teleoperation framework for humanoid loco-manipulation: An application for the icub robot," IEEE Robot. Autom. Mag., vol. 26, no. 4, pp. 73-82, 2019.

[20] M. Talha et al., "Towards robotic decommissioning of legacy nuclear plant: Results of human-factors experiments with tele-robotic manipulation, and a discussion of challenges and approaches for decommissioning," in Proc. IEEE Int. Symp. on Safety, Security, and Rescue Robotics, 2016, pp. 166-173.

[21] D. Lee et al., "Semiautonomous haptic teleoperation control architecture of multiple unmanned aerial vehicles," IEEE/ASME Trans. Mechatron., vol. 18, no. 4, pp. 1334-1345, 2013.

[22] B. D. Argall, "Autonomy in rehabilitation robotics: An intersection," Annual Review of Control, Robotics, and Autonomous Systems, vol. 1, no. 1, pp. 441-463, 2018.

[23] L. Xiong et al., "Shared control of a medical robot with haptic guidance," Int J Comput Assist Radiol Surg, vol. 12, no. 1, pp. 137-147, 2017.

[24] M. Young et al., "Formalized task characterization for human-robot autonomy allocation," in Proc. Int. Conf. on Robot. and Autom., 2019, pp. 6044-6050.
[25] F. Abi-Farraj et al., "A visual-based shared control architecture for remote telemanipulation," in Proc. IEEE/RSJ Int. Conf. Intell. Robots Syst., 2016, pp. 4266-4273.

[26] J. Colgate et al., "Cobots: robots for collaboration with human operators," in Proc. ASME International Mechanical Engineering Congress and Exposition, 1996, pp. 433-439.

[27] S. Haddadin et al., Physical Human-Robot Interaction. Cham: Springer International Publishing, 2016, pp. 1835-1874.

[28] T. Takubo et al., "Human-robot cooperative manipulation using a virtual nonholonomic constraint," Int J Rob Res, vol. 21, no. 5-6, pp. 541-553, 2002.

[29] A. Mörtl et al., "The role of roles: Physical cooperation between humans and robots," Int J Rob Res, vol. 31, no. 13, pp. 1656-1674, 2012.

[30] J. Arnold et al., "Variable impedance control for phri: Impact on stability, agility, and human effort in controlling a wearable ankle robot," IEEE Robot. Autom. Lett., vol. 6, no. 2, pp. 2429-2436, 2021.

[31] S. A. Bowyer et al., "Active constraints/virtual fixtures: A survey," IEEE Trans. Robot., vol. 30, no. 1, pp. 138-157, 2014.

[32] E. Magrini et al., "Hybrid force/velocity control for physical humanrobot collaboration tasks," in Proc. IEEE/RSJ Int. Conf. Intell. Robots Syst., 2016, pp. 857-863.

[33] S. Tarbouriech et al., "Admittance control for collaborative dual-arm manipulation," in Proc. Int. Conf. on Advanced Robotics, 2019, pp. 198-204.

[34] Y. Hirata et al., "Distributed robot helpers handling a single object in cooperation with a human," in Proc. IEEE Int. Conf. Robot. Autom., vol. 1,2000 , pp. $458-463$.

[35] M. Lawitzky et al., "Load sharing in human-robot cooperative manipulation," in 19th Int. Symp. in Robot and Human Int. Comm., 2010, pp. $185-191$.

[36] M. Lippi et al., "Human multi-robot physical interaction: a distributed framework," J. Intell. Robot. Syst., vol. 101, no. 2, p. 35, 2021.

[37] A. Nguyen et al., "Driver-automation cooperative approach for shared steering control under multiple system constraints: Design and experiments," IEEE Trans. Ind. Electron., vol. 64, no. 5, pp. 3819-3830, 2017.

[38] J. Alonso-Mora et al., "Shared control of autonomous vehicles based on velocity space optimization," in Proc. IEEE Int. Conf. Robot. Autom. 2014, pp. 1639-1645.

[39] M. Selvaggio et al., "Passive task-prioritized shared-control teleoperation with haptic guidance," in Proc. Int. Conf. Robot. Autom., 2019, pp. $430-436$.

[40] G. Lentini et al., "Alter-ego: A mobile robot with a functionally anthropomorphic upper body designed for physical interaction," IEEE Robot Autom Mag, vol. 26, no. 4, pp. 94-107, 2019.

[41] C. Masone et al., "Shared planning and control for mobile robots with integral haptic feedback," Int J Rob Res, vol. 37, no. 11, pp. 1395-1420, 2018.

[42] M. Cognetti et al., "Perception-aware human-assisted navigation of mobile robots on persistent trajectories," IEEE Robot. Autom. Lett., vol. 5, no. 3, pp. 4711-4718, 2020

[43] T. M. Alexander Broad et al., "Highly parallelized data-driven mpc for minimal intervention shared control," in Proc. Robotics: Science and Systems, 2019.

[44] M. Rubagotti et al., "Semi-autonomous robot teleoperation with obstacle avoidance via model predictive control," IEEE Robot. Autom. Lett., vol. 4, no. 3, pp. 2746-2753, 2019.

[45] S. Hayati et al., "Dual arm coordination and control," Robot. Auton. Syst., vol. 5, no. 4, pp. 333-344, 1989.

[46] M. Laghi et al., "Shared-autonomy control for intuitive bimanual telemanipulation," in IEEE-RAS 18th Int. Conf. on Humananoid Robots, 2018, pp. 1-9.

[47] A. Hernansanz et al., "A multi-robot cooperation strategy for dexterous task oriented teleoperation," Robotics and Autonomous Systems, vol. 68, pp. 156-172, 2015.

[48] D. Nicolis et al., "Occlusion-free visual servoing for the shared autonomy teleoperation of dual-arm robots," IEEE Robot. Autom. Lett., vol. 3, no. 2, pp. 796-803, 2018.

[49] M. Selvaggio et al., "Haptic-based shared-control methods for a dualarm system," IEEE Robot. Autom. Lett., vol. 3, no. 4, pp. 4249-4256, 2018.

[50] A. Franchi et al., "Shared control: Balancing autonomy and human assistance with a group of quadrotor uavs," IEEE Robot Autom Mag, vol. 19 , no. 3, pp. 57-68, Sep. 2012. 
[51] _ - "Bilateral teleoperation of groups of mobile robots with timevarying topology," IEEE Trans. Robot., vol. 28, no. 5, pp. 1019-1033, 2012.

[52] G. Gioioso et al., "The flying hand: A formation of uavs for cooperative aerial tele-manipulation," in Proc. IEEE Int. Conf. Robot. Autom., 2014, pp. $4335-4341$.

[53] M. Mohammadi et al., "Cooperative aerial tele-manipulation with haptic feedback," in Proc. IEEE/RSJ Int. Conf. Intell. Robots Syst., 2016, pp. 5092-5098.

[54] N. Staub et al., "The tele-magmas: An aerial-ground comanipulator system," IEEE Robot Autom Mag, vol. 25, no. 4, pp. 66-75, 2018.

[55] S. Musić et al., "Control sharing in human-robot team interaction," Annual Reviews in Control, vol. 44, pp. 342 - 354, 2017.

[56] P. Berthet-Rayne et al., "Hubot: A three state human-robot collaborative framework for bimanual surgical tasks based on learned models," in Proc. IEEE Int. Conf. Robot. Autom., 2016, pp. 715-722.

[57] A. Pichler et al., "Towards shared autonomy for robotic tasks in manufacturing," Procedia Manufacturing, vol. 11, pp. 72 - 82, 2017.

[58] Y. Gao et al., "Review on space robotics: Toward top-level science through space exploration," Sci. Robot., vol. 2, no. 7, 2017.

[59] S. Nikolaidis et al., "Human-robot mutual adaptation in shared autonomy," in Proc. ACM/IEEE Int. Conf. on Hum.-Robot Interact., 2017, pp. 294-302.

[60] — "Human-robot mutual adaptation in collaborative tasks: Models and experiments," Int J Rob Res, vol. 36, no. 5-7, pp. 618-634, 2017.

[61] A. D. Dragan et al., "A policy-blending formalism for shared control," Int J Rob Res, vol. 32, no. 7, pp. 790-805, 2013.

[62] D. Gopinath et al., "Human-in-the-loop optimization of shared autonomy in assistive robotics," IEEE Robot. Autom. Lett., vol. 2, no. 1, pp. 247-254, 2016.

[63] H. J. Jeon et al., "Shared autonomy with learned latent actions," arXiv preprint arXiv:2005.03210, 2020

[64] D. Rakita et al., "Shared control-based bimanual robot manipulation," Sci. Robot., vol. 4, no. 30, 2019.

[65] Y. Li et al., "Continuous role adaptation for human-robot shared control," IEEE Trans. Robot., vol. 31, no. 3, pp. 672-681, 2015.

[66] _-, "A framework of human-robot coordination based on game theory and policy iteration," IEEE Trans. Robot., vol. 32, no. 6, pp. 1408-1418, 2016.

[67] L. Peternel et al., "Robot adaptation to human physical fatigue in human-robot co-manipulation," Auton. Robots, vol. 42, p. 1011-1021, 2018.

[68] R. Rahal et al., "Caring about the human operator: Haptic shared control for enhanced user comfort in robotic telemanipulation," IEEE Trans. Haptics, vol. 13, no. 1, pp. 197-203, 2020.

[69] Q. Ji et al., "A probabilistic framework for modeling and real-time monitoring human fatigue," IEEE Trans. Syst., Man, Cybern. A, Syst. Humans, vol. 36, no. 5, pp. 862-875, 2006.

[70] N. Enayati et al., "Skill-based human-robot cooperation in teleoperated path tracking," Auton. Robots, vol. 42, no. 5, pp. 997-1009, 2018.

[71] N. Enayati et al., "Robotic assistance-as-needed for enhanced visuomotor learning in surgical robotics training: An experimental study," in Proc. IEEE Int. Conf. Robot. Autom., 2018, pp. 6631-6636.

[72] L. Milliken et al., "Modeling user expertise for choosing levels of shared autonomy," in Proc. IEEE Int. Conf. Robot. Autom., 2017, pp. $2285-2291$.

[73] J. Trafton et al., "Enabling effective human-robot interaction using perspective-taking in robots," IEEE Trans. Syst., Man, Cybern. A, vol. 35 , no. 4 , pp. $460-470,2005$.

[74] M. Berlin et al., "Perspective taking: An organizing principle for learning in human-robot interaction," in Proc. 21 st National Conference on Artificial Intelligence - Vol. 2. AAAI Press, 2006, p. 1444-1450.

[75] G. Milliez et al., "A framework for endowing an interactive robot with reasoning capabilities about perspective-taking and belief management," in IEEE Int. Symp. on Robot and Human Interactive Communication, 2014, pp. 1103-1109.

[76] B. Scassellati, "Theory of mind for a humanoid robot," Auton. Robots, vol. 12 , no. 1, pp. 13-24, Jan 2002

[77] S. Devin et al., "An implemented theory of mind to improve humanrobot shared plans execution," in 2016 ACM/IEEE Int. Conf. Hum.Robot Interact., 2016, pp. 319-326.

[78] N. Rabinowitz et al., "Machine theory of mind," in Proc. Int. Conf. on Machine Learning, ser. Proceedings of Machine Learning Research, J. Dy et al., Eds., vol. 80. PMLR, 2018, pp. 4218-4227.
[79] F. Abi-Farraj et al., "A learning-based shared control architecture for interactive task execution," in Proc. IEEE Int. Conf. Robot. Autom., 2017, pp. 329-335.

[80] I. Havoutis et al., "Learning assistive teleoperation behaviors from demonstration," in IEEE Int. Symp. on Safety, Security, and Rescue Robotics, 2016, pp. 258-263.

[81] — "Supervisory teleoperation with online learning and optimal control," in Proc. IEEE Int. Conf. Robot. Autom., 2017, pp. 1534-1540.

[82] I. Havoutis et al., "Learning from demonstration for semi-autonomous teleoperation," Auton. Robots, vol. 43, no. 3, pp. 713-726, 2019.

[83] C. J. P. del Pulgar et al., "Using learning from demonstration to generate real-time guidance for haptic shared control," in Proc. IEEE Int. Conf. Syst. Man. Cybern., 2016, pp. 3205-3210.

[84] R. Balachandran et al., "Adaptive authority allocation in shared control of robots using bayesian filters," in Proc. IEEE Int. Conf. Robot. Autom., 2020, pp. 11 298-11304

[85] M. K. O'Malley et al., "Shared Control in Haptic Systems for Performance Enhancement and Training," J Dyn Syst Meas Control, vol. 128, no. 1, pp. 75-85, 112005

[86] J. Luo et al., "A teleoperation framework for mobile robots based on shared control," IEEE Robot. Autom. Lett., vol. 5, no. 2, pp. 377-384, 2020.

[87] L. B. Rosenberg, "Virtual fixtures: Perceptual tools for telerobotic manipulation," in Proc. IEEE Virtual Reality Annual Int. Symp., 1993, pp. 76-82.

[88] A. Munawar et al., "A surgical robot teleoperation framework for providing haptic feedback incorporating virtual environment-based guidance," Front. Robot. AI, vol. 3, p. 47, 2016.

[89] A. Ghalamzan E. et al., "Human-in-the-loop optimisation: Mixed initiative grasping for optimally facilitating post-grasp manipulative actions," in Proc. IEEE/RSJ Int. Conf. Intell. Robots Syst., 2017, pp. 3386-3393.

[90] S. Parsa et al., "Haptic-guided shared control grasping: collision-free manipulation," in Proc. IEEE 16th Int. Conf. on Automation Science and Engineering, 2020, pp. 1552-1557.

[91] M. Selvaggio et al., "Haptic-guided shared control for needle grasping optimization in minimally invasive robotic surgery," in Proc. IEEE/RSJ Int. Conf. Intell. Robots Syst., 2019, pp. 3617-3623.

[92] J. Ramos et al., "Dynamic locomotion synchronization of bipedal robot and human operator via bilateral feedback teleoperation," Sci. Robot., vol. 4, no. 35, 2019.

[93] M. J. A. Zeestraten et al., "Programming by demonstration for shared control with an application in teleoperation," IEEE Robot. Autom. Lett., vol. 3, no. 3, pp. 1848-1855, July 2018.

[94] D. Aarno et al., "Adaptive virtual fixtures for machine-assisted teleoperation tasks," in Proc. IEEE Int. Conf. Robot. Autom., 2005, pp. 1139-1144.

[95] S. Ekvall et al., "Online task recognition and real-time adaptive assistance for computer-aided machine control," IEEE Trans. Robot., vol. 22, no. 5, pp. 1029-1033, 2006.

[96] S. Javdani et al., "Shared autonomy via hindsight optimization for teleoperation and teaming," Int J Rob Res, vol. 37, no. 7, pp. 717-742, 2018.

[97] M. Chen et al., "Trust-aware decision making for human-robot collaboration: Model learning and planning," J. Hum.-Robot Interact., vol. 9, no. $2,2020$.

[98] R. Luo et al., "A workload adaptive haptic shared control scheme for semi-autonomous driving," Accid. Anal. Prev., vol. 152, p. 105968 , 2021.

[99] K. S. Welfare et al., "Consider the human work experience when integrating robotics in the workplace," in Proc. ACM/IEEE Int. Conf. on Hum.-Robot Interact., 2019, pp. 75-84.

[100] P. A. Lasota et al., A survey of methods for safe human-robot interaction. Now Publishers, 2017

[101] P. Robuffo Giordano et al., "A passivity-based decentralized strategy for generalized connectivity maintenance," Int J Rob Res, vol. 32, no. 3 , pp. 299-323, 2013.

[102] S. Reddy et al., "Shared autonomy via deep reinforcement learning," in Proc. Robotics: Science and Systems, 2018.

[103] I. Gaudiello et al., "Trust as indicator of robot functional and social acceptance. an experimental study on user conformation to icub answers," Computers in Human Behavior, vol. 61, pp. 633-655, 2016.

[104] V. Alonso et al., "System transparency in shared autonomy: A mini review," Front. Neurorobot., vol. 12, p. 83, 2018.

[105] M. Fontaine et al., "A quality diversity approach to automatically generating human-robot interaction scenarios in shared autonomy," arXiv preprint arXiv:2012.04283, 2020. 